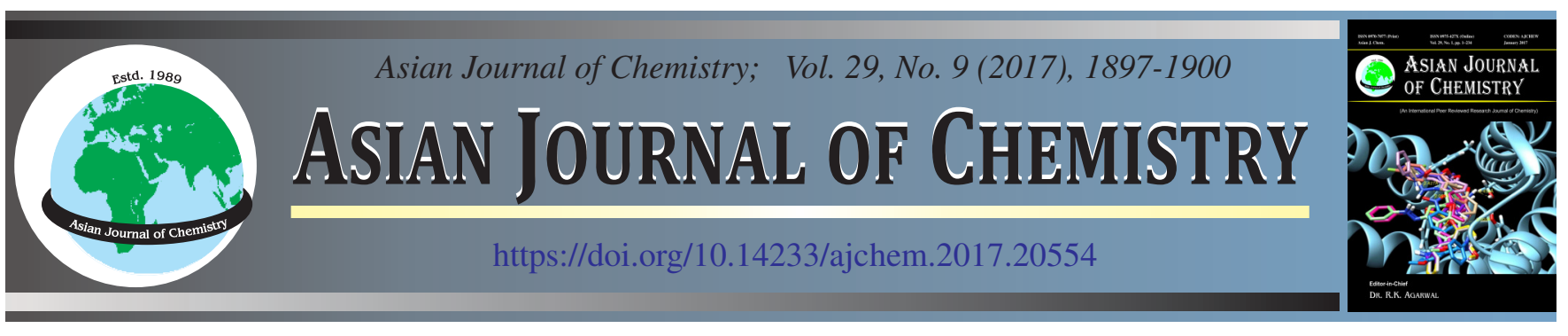

\title{
Determination and Assay of Some Antineoplastic Agents in Pure Form and in their Pharmaceutical Preparation
}

\author{
I.C. ShuKLa*, O.P. YadAV*, SAnJai Kumar and Virendra Kumar
}

Department of Chemistry, University of Allahabad, Allahabad-211 002, India

*Corresponding authors: E-mail: prof.icshukla@rediffmail.com; opau09@gmail.com

Keywords: Antineoplastic agents, Titration, Standard deviation, Coefficient of variation.

\section{INTRODUCTION}

Antineoplastic agents are antimetabolite and well known for exhibiting a wide range of pharmacological and biological activities. They check the metabolism of neoplasmic cells either by converting or binding the metabolic product of neoplasmic cells. Levamisole, (6-phenyl-2,3,5,6-tetrahydroimidazo[2,1b]thiazole) is an anthelmintic and immunomedulator. It has been used in humans to treat parasitic worm infections [1] and has been studied in combination with other form of chemotherapy for colon cancer, melanoma and head and neck cancer [2]. Promethazine hydrochloride (10-[(2-dimethyl-amino)propyl] phenothiazine hydrochloride) is long acting antihistaminic, antagonize, the vasodilator and vasoconstrictor action of histamine [3]. Daily dose of promethazine hydrochloride required to maintain an effect on intraderminal injection of histamine in man [3]. Letrozole (4,4'-(1H-1,2,4-triazol-1-yl)methylene dibenzonitrile) is an oral non-steroidal aromataze inhibitor for the treatment of hormonally-responsive breast cancer after surgery and also used for the treatment of gynecomastia [4]. Chloropromazine hydrochloride (3-(2-chloro-10H-phenothiazine-10-yl)-N,N-dimethylpropane-1-amine) has widely been used in the treatment of schizophrenia and related disorder [5]. Fosfestrol ([4-\{4-(4-phasphono-oxyphenyl)hex-3-en-3yl phenoxy]phosphonic acid) is a synthetic estrogen used as an antineoplastic agent. Fosfestrol tetrasodium is an inactive synthetic estrogen which is converted in body to an active estrogen diethylstilbestrol and used in the treatment of prostate cancer [6]. Mitomycin ([6-amino-8a-methoxy-5-methyl-4,7dioxo-1,1a,2,4,7,8,8a,8b-octahydroazireno[2',3',3,4]pyrrolo$(1,2 \mathrm{a})$ indol-8-yl] methyl carbamate) is in the family of aziridine containing natural product. It is used to treat upper gastrointestinal (esophageal carcinoma), anal and breast cancer [7].

Due to the biological and pharmacological importance of these drugs several methods [8-10] were reported for quantitative evaluation of these antineoplastic agents. Most of the methods use sophisticated instruments like HPLC [11], TLC [12], colorimetry [13], infrared spectrophotometry [14] and other techniques. Herein, we reported a simple titrimetric method for assay of some antineoplastic drugs i.e. chloropromethazine hydrochloride, promethazine hydrochloride, levamisole, fosfestrol, letrozole, and mitomycin by using N-bromosuccinimide reagent in acidic medium. This reagent [15-19] has been used for allylic or benzylic bromination, oxidation reaction 
for primary and secondary alcohol, aromatization reaction and addition to olefinic double bonds. Present method may easily be adopted in any pharmaceutical laboratory having no sophisticated instruments. To establish the authenticity of the method recovery experiments were also done by standard drug addition method.

\section{EXPERIMENTAL}

N-Bromosuccinimide (NBS) 0.02N (Loba Chemie) was prepared by dissolving accurately weighed $0.356 \mathrm{~g}$ of N-bromosuccinimide in $100 \mathrm{~mL}$ volumetric flask. Sodium thiosulphate solution $(0.02 \mathrm{~N})$ (Merck) was prepared in $100 \mathrm{~mL}$ distilled water in a volumetric flask. $\mathrm{CuSO}_{4} \cdot 5 \mathrm{H}_{2} \mathrm{O}(0.02 \mathrm{~N})$ (Qualigens) solution was prepared in distilled water in a $100 \mathrm{~mL}$ volumetric flask, potassium iodide $5 \% \mathrm{w} / \mathrm{v}$ (Qualigens) aqueous solution, $1 \% \mathrm{w} / \mathrm{v}$ starch solution (Merck) and glacial acetic acid (4 N) (Merck) were also prepared.

Accurately weighed $(100 \mathrm{mg}$ ) pure sample of chloropromazine hydrochloride, promathazine hydrochloride, levamisole, letrozole, and mitomycin were dissolved in distilled water and fosfestrol was dissolve in ethanol in a $100 \mathrm{~mL}$ volumetric flask and solution made up to the mark to give a concentration of $1 \mathrm{mg} / \mathrm{mL}$.

Twenty tablets of pharmaceutical products were crushed to a fine powder and powder equivalent to $100 \mathrm{mg}$ of sample was taken in $100 \mathrm{~mL}$ calibrated volumetric flask and dissolved similarly. No residue was noted in any of the sample. As described above, the contents of the injection equivalent to 50 $\mathrm{mg}$ of the pure sample were taken to get a concentration of $1 \mathrm{mg} / \mathrm{mL}$.

General procedure: Aliquots containing 1-5 mg of the sample were taken in $100 \mathrm{~mL}$ stoppered conical flask and $5 \mathrm{~mL}$ of $0.02 \mathrm{~N} \mathrm{~N}$-bromosuccinimide and $5 \mathrm{~mL}$ of $4 \mathrm{~N}$ acetic acid was added to it. The reaction mixture was shaken well and allowed to react for required reaction time $(10 \mathrm{~min})$ at room temperature $\left(25-30{ }^{\circ} \mathrm{C}\right)$. After the reaction was over $5 \mathrm{~mL}$ of $5 \%$ potassium iodide solution was added to it, content shaken thoroughly and allowed to stand for a minute. The liberated iodine was titrated against $0.02 \mathrm{~N}$ sodium thiosulphate solution using starch indicator. A blank experiment was also done under identical condition using all the reagents except sample. The amount of sample was calculated by the difference in titre values of $0.02 \mathrm{~N}$ sodium thiosulphate solution used for actual and blank experiments. On the basis of percentage error, the value of SD and coefficient of variation were also calculated (Table-1). The same procedure was applied for the determination of pharmaceutical preparations. Excipients present in pharmaceutical preparations do not interfere.

\section{RESULTS AND DISCUSSION}

Taking chloropromazine hydrochloride as a test sample the stoichiometric ratio with the reagent was established. It was observed that three moles of the reagent were needed for complete reaction. Similarly for other compounds also the stoichiometry was determined it was found that the ratio between $\mathrm{N}$-bromosuccinimide reagent and antineoplastic agents varies. e.g. levamisole $(2: 1)$, promazine hydrochloride $(3: 1)$, letrozole $(3: 1)$, fosfestrol $(2: 1)$ and mitomycin $(3: 1)$. The ratio remains constant even under varying reaction conditions i.e. change in reaction time, concentration of the reagent, reaction medium, reaction temperature etc. It was observed that a lesser reaction time $(<10 \mathrm{~min})$ gives inaccurate results because of incomplete reaction. Increase in the reaction time (15-20 min) does not change percentage recovery of the sample. As compared to sulphuric acid and nitric acid the use of acetic acid was found as a proper reaction medium. Reaction was also carried out in the absence of acetic acid and it was found that the results were very low because of incomplete dissociation of the reagent. The concentration effect of acetic acid was also studied and $10 \mathrm{~mL}$ of $4 \mathrm{~N}$ acetic acid was found suitable for accurate results.

$\mathrm{N}$-Bromosuccinimide is the main reactive species with all the compounds, therefore variation in its concentration was essential. While studying various $(0.01-1.0 \mathrm{~N})$ concentrations it was found that $0.02 \mathrm{~N}$ NBS was sufficient for accurate result. With variable volume of NBS $(1-10 \mathrm{~mL})$ it was observed that $5 \mathrm{~mL}$ reagent of $0.02 \mathrm{~N}$ concentration gives accurate result. Higher concentration (0.03-1.0 N) and volume (6-10 mL) does not improve results. The effect of reaction temperature has also been studied. It was observed that the reaction is very slow at ice cold temperature $\left(0-5{ }^{\circ} \mathrm{C}\right)$. As indicated in Table- 1 the best results were obtained at room temperature $\left(25-30^{\circ} \mathrm{C}\right)$. Increase in reaction temperature above $\left(30-100^{\circ} \mathrm{C}\right)$ gives in accurate results because of decomposition of the reagent. On increasing the reaction time (15-30 min) there was no effect on the percentage recovery. Results obtained at standard reaction conditions are reported in Table- 1 . To verify the precision of the method recovery experiments were done by standard drug addition method (Table-2). It shows that the suggested method is accurate, reproducible and precise. It can easily be adopted in an ordinary pharmaceutical laboratory where sophisticated instruments are not available. Indian pharmacopeia does not describe such type of method for the determination of compounds under study. Only chlorpromazine hydrochloride, promethazine hydrochloride and levamisole have been determined by infrared spectrophotometry and thin layer chromatography in Indian pharmacopeia [20].

\section{Calculation}

For each experiment, the amount of sample was calculated by following expression:

$$
\text { Weight of sample }(\mathrm{mg})=\frac{\mathrm{M} \times \mathrm{N}(\mathrm{B}-\mathrm{S})}{\mathrm{n}}
$$

where, $\mathrm{M}=$ molecular weight of the sample, $\mathrm{N}=$ normality of sodium thiosulphate solution, $\mathrm{B}=$ volume of sodium thiosulphate solution for blank, $\mathrm{S}=$ volume of sodium thiosulphate solution for sample, $\mathrm{n}=$ stoichiometry of the reaction.

For testing authenticity of the recommended procedure standard deviation (SD) and coefficient of variation (CV) were also calculated. At least nine determinations were carried out and the results noted. The proposed method was further justified by recovery experiments through standard drug addition method. A known amount of the pure compound was taken and to this, varying amounts of pharmaceutical product of the same compounds were added. The total amount of the sample was found by the following expression: 


\begin{tabular}{|c|c|c|c|c|c|c|c|c|}
\hline \multicolumn{9}{|c|}{$\begin{array}{l}\text { TABLE-1 } \\
\text { MILLIGRAM DETERMINATION OF SOME ANTINEOPLASTIC AGENTS IN PURE FORM AND IN } \\
\text { THEIR PHARMACEUTICAL PREPARATIONS WITH }(0.02 \mathrm{~N}) \text { NBS REAGENT IN ACIDIC MEDIUM }\end{array}$} \\
\hline Sample & $\begin{array}{c}\text { Aliquots } \\
\text { taken (mL) }\end{array}$ & $\begin{array}{c}\text { Amount } \\
\text { present* }(\mathrm{mg})\end{array}$ & $\begin{array}{l}\text { Reaction } \\
\text { time (min) }\end{array}$ & $\begin{array}{l}\text { Molecularity } \\
\text { (n) }\end{array}$ & $\begin{array}{l}\text { Amount obtained } \\
\text { by calculation*** }\end{array}$ & Error $(\%)$ & SD & $\mathrm{CV}$ \\
\hline \multirow{3}{*}{$\begin{array}{l}\text { Chloropromzine } \\
\text { hydrochloride (Pure) }\end{array}$} & 1 & 0.998 & 10 & 2 & 0.989 & -0.90 & 0.0039 & 0.3943 \\
\hline & 3 & 2.995 & 10 & 2 & 2.974 & -0.70 & 0.0026 & 0.0874 \\
\hline & 5 & 4.994 & 10 & 2 & 4.964 & -0.50 & 0.0017 & 0.0342 \\
\hline \multirow{3}{*}{ Relitil-25 (Tablet) } & 1 & 0.962 & 10 & 2 & 0.953 & -0.93 & 0.0040 & 0.4197 \\
\hline & 3 & 2.884 & 10 & 2 & 2.862 & -0.76 & 0.0031 & 0.1083 \\
\hline & 5 & 4.810 & 10 & 2 & 4.784 & -0.54 & 0.0027 & 0.0564 \\
\hline \multirow{3}{*}{$\begin{array}{l}\text { Promazine } \\
\text { hydrochloride (Pure) }\end{array}$} & 1 & 0.964 & 10 & 3 & 0.951 & -1.65 & 0.0034 & 0.3574 \\
\hline & 3 & 2.887 & 10 & 3 & 2.859 & -0.96 & 0.0028 & 0.0979 \\
\hline & 5 & 4.815 & 10 & 3 & 4.789 & -0.53 & 0.0024 & 0.0501 \\
\hline \multirow{3}{*}{ Phenergan (Tablet) } & 1 & 0.965 & 10 & 3 & 0.956 & -0.93 & 0.0029 & 0.3033 \\
\hline & 3 & 2.900 & 10 & 3 & 2.872 & -0.96 & 0.0023 & 0.0800 \\
\hline & 5 & 4.837 & 10 & 3 & 4.809 & -0.57 & 0.0018 & 0.0374 \\
\hline \multirow{3}{*}{ Phenergan (Injection) } & 1 & 0.962 & 10 & 3 & 0.948 & -1.45 & 0.0021 & 0.2215 \\
\hline & 3 & 2.908 & 10 & 3 & 2.892 & -0.55 & 0.0017 & 0.0588 \\
\hline & 5 & 4.800 & 10 & 3 & 4.789 & -0.22 & 0.0021 & 0.0438 \\
\hline \multirow{3}{*}{ Levamisole (Pure) } & 1 & 0.989 & 10 & 2 & 0.968 & -1.12 & 0.0025 & 0.2582 \\
\hline & 3 & 2.945 & 10 & 2 & 2.908 & -1.25 & 0.0022 & 0.0756 \\
\hline & 5 & 4.898 & 10 & 2 & 4.872 & -0.53 & 0.0019 & 0.0390 \\
\hline \multirow{3}{*}{ Devormis (Tablet) } & 1 & 0.982 & 10 & 2 & 0.972 & -1.01 & 0.0041 & 0.4218 \\
\hline & 3 & 2.945 & 10 & 2 & 2.916 & -0.98 & 0.0036 & 0.1234 \\
\hline & 5 & 4.902 & 10 & 2 & 4.870 & -0.65 & 0.0031 & 0.0637 \\
\hline \multirow{3}{*}{ Dicaris (Tablet) } & 1 & 0.974 & 10 & 2 & 0.964 & -1.02 & 0.0028 & 0.2905 \\
\hline & 3 & 2.920 & 10 & 2 & 2.896 & -0.82 & 0.0024 & 0.2890 \\
\hline & 5 & 4.864 & 10 & 2 & 4.830 & -0.69 & 0.0019 & 0.0393 \\
\hline \multirow{3}{*}{ Levamole (Tablet) } & 1 & 0.978 & 10 & 2 & 0.965 & -1.32 & 0.0032 & 0.3316 \\
\hline & 3 & 2.932 & 10 & 2 & 2.914 & -0.61 & 0.0028 & 0.0960 \\
\hline & 5 & 4.894 & 10 & 2 & 4.868 & -0.53 & 0.0021 & 0.0431 \\
\hline \multirow{3}{*}{ Vermisole (Tablet) } & 1 & 0.973 & 10 & 2 & 0.962 & -1.13 & 0.0025 & 0.2599 \\
\hline & 3 & 2.917 & 10 & 2 & 2.895 & -0.75 & 0.0021 & 0.0725 \\
\hline & 5 & 4.862 & 10 & 2 & 4.837 & -0.51 & 0.0015 & 0.0310 \\
\hline \multirow{3}{*}{ Fosfestrol (Pure) } & 1 & 0.969 & 10 & 2 & 0.959 & -1.03 & 0.0020 & 0.2038 \\
\hline & 3 & 2.908 & 10 & 2 & 2.885 & 0.79 & 0.0016 & 0.0554 \\
\hline & 5 & 4.846 & 10 & 2 & 4.818 & -0.57 & 0.0010 & 0.0207 \\
\hline \multirow{3}{*}{ Honven (Tablet) } & 1 & 0.954 & 10 & 2 & 0.944 & -1.05 & 0.0018 & 0.1907 \\
\hline & 3 & 2.863 & 10 & 2 & 2.841 & -0.76 & 0.0017 & 0.0634 \\
\hline & 5 & 4.771 & 10 & 2 & 4.748 & -0.48 & 0.0031 & 0.0358 \\
\hline \multirow{3}{*}{ Letrozole (Pure) } & 1 & 0.964 & 10 & 3 & 0.934 & -1.05 & 0.0022 & 0.3854 \\
\hline & 3 & 2.832 & 10 & 3 & 2.811 & -0.74 & 0.0029 & 0.0961 \\
\hline & 5 & 4.720 & 10 & 3 & 4.695 & -0.53 & 0.0030 & 0.0660 \\
\hline \multirow{3}{*}{ Lupride (Tablet) } & 1 & 0.884 & 10 & 3 & 0.878 & -1.01 & 0.0016 & 0.1822 \\
\hline & 3 & 2.661 & 10 & 3 & 2.693 & -0.83 & 0.0012 & 0.0455 \\
\hline & 5 & 4.435 & 10 & 3 & 4.412 & -0.52 & 0.0014 & 0.0317 \\
\hline & 1 & 0.923 & 10 & 3 & 0.913 & -1.02 & 0.0022 & 0.2410 \\
\hline Progtage (Tablet) & 3 & 2.769 & 10 & 3 & 2.749 & -0.72 & 0.0024 & 0.0873 \\
\hline & 5 & 4.615 & 10 & 3 & 4.591 & -0.52 & 0.0030 & 0.0653 \\
\hline & 1 & 0.991 & 10 & 3 & 0.981 & -1.00 & 0.0076 & 0.7447 \\
\hline Mitomycin (Pure) & 3 & 2.973 & 10 & 3 & 2.951 & -0.73 & 0.0051 & 0.1728 \\
\hline & 5 & 4.955 & 10 & 3 & 4.932 & -0.46 & 0.0035 & 0.0710 \\
\hline & 1 & 0.936 & 10 & 3 & 0.926 & -0.99 & 0.0042 & 0.4536 \\
\hline Almito (Injection) & 3 & 2.808 & 10 & 3 & 2.784 & -0.86 & 0.0038 & 0.1365 \\
\hline & 5 & 4.680 & 10 & 3 & 4.651 & -0.62 & 0.0026 & 0.0559 \\
\hline & 1 & 0.964 & 10 & 3 & 0.952 & -1.24 & 0.0021 & 0.2206 \\
\hline Mitocin (Vial) & 3 & 2.892 & 10 & 3 & 2.868 & -0.83 & 0.0078 & 0.2720 \\
\hline & 5 & 4.820 & 10 & 3 & 4.802 & -0.37 & 0.0096 & 0.1999 \\
\hline & 1 & 0.986 & 10 & 3 & 0.972 & -1.32 & 0.0067 & 0.6893 \\
\hline Oncicine (Tablet) & 3 & 2.946 & 10 & 3 & 2.916 & -0.71 & 0.0062 & 0.2111 \\
\hline & 5 & 4.930 & 10 & 3 & 4.910 & -0.41 & 0.0081 & 0.1650 \\
\hline
\end{tabular}

*In each sample nine determinations were done; **Average of nine determinations. 


\begin{tabular}{|c|c|c|c|c|c|c|c|c|c|}
\hline \multicolumn{10}{|c|}{$\begin{array}{c}\text { TABLE-2 } \\
\text { RECOVERY STUDIES BY STANDARD DRUG ADDITION METHOD }\end{array}$} \\
\hline Name of drugs & $\begin{array}{l}\text { S. } \\
\text { No. }\end{array}$ & $\begin{array}{l}\text { Number of } \\
\text { observation } \\
\text { (N) }\end{array}$ & $\begin{array}{c}\text { Amount } \\
\text { present } \\
\text { (Pure) } \\
\text { (mg) }\end{array}$ & $\begin{array}{c}\text { Amount of } \\
\text { drug added } \\
(\mathrm{mg}){ }^{\prime} \mathrm{X}\end{array}$ & $\begin{array}{c}\text { Total } \\
\text { amount of } \\
\text { drug } \\
\text { obtained by } \\
\text { calculation } \\
(\mathrm{mg})\end{array}$ & $\begin{array}{l}\text { Amount of } \\
\text { drug } \\
\text { obtained by } \\
\text { calculation } \\
(\mathrm{mg}) \text { ' } \mathrm{Y} \text { ' }\end{array}$ & $\mathrm{XY}$ & $X^{2}$ & $\begin{array}{l}\text { Recovery } \\
(\%)\end{array}$ \\
\hline \multirow{5}{*}{$\begin{array}{l}\text { Chloropromazine } \\
\text { hydrochloride }\end{array}$} & 1 & 3 & 0.998 & 0.692 & 1.942 & 0.953 & 0.918 & 0.925 & \multirow{4}{*}{99.07} \\
\hline & 2 & 3 & 0.998 & 1.924 & 2.895 & 1.906 & 3.667 & 3.702 & \\
\hline & 3 & 3 & 0.998 & 2.886 & 3.848 & 2.859 & 8.251 & 8.329 & \\
\hline & 4 & 3 & 0.998 & 3.848 & 4.801 & 3.812 & 14.669 & 14.807 & \\
\hline & & $\Sigma \mathrm{N}=12$ & & $\Sigma X=9.620$ & & $\Sigma Y=9.526$ & $\Sigma X Y=27.501$ & $\Sigma X^{2}=27.793$ & \multirow{5}{*}{98.16} \\
\hline \multirow{5}{*}{$\begin{array}{l}\text { Promathazine } \\
\text { Hydrochloride }\end{array}$} & 1 & 3 & 0.967 & 0.965 & 1.907 & 0.956 & 0.923 & 0.931 & \\
\hline & 2 & 3 & 0.967 & 1.930 & 2.863 & 1.912 & 3.690 & 3.725 & \\
\hline & 3 & 3 & 0.967 & 2.895 & 3.819 & 2.869 & 8.120 & 8.381 & \\
\hline & 4 & 3 & 0.967 & 3.860 & 4.775 & 3.824 & 14.761 & 14.900 & \\
\hline & & $\Sigma \mathrm{N}=12$ & & $\Sigma X=9.650$ & & $\Sigma Y=9.560$ & $\Sigma X Y=27.494$ & $\Sigma X^{2}=27.937$ & \multirow{5}{*}{98.58} \\
\hline \multirow{5}{*}{ Levamisole } & 1 & 3 & 0.989 & 0.982 & 1.940 & 0.968 & 0.951 & 0.964 & \\
\hline & 2 & 3 & 0.989 & 1.964 & 2.908 & 1.936 & 3.802 & 3.857 & \\
\hline & 3 & 3 & 0.989 & 2.946 & 3.876 & 2.904 & 8.555 & 8.679 & \\
\hline & 4 & 3 & 0.989 & 3.928 & 4.844 & 3.972 & 15.209 & 15.429 & \\
\hline & & $\Sigma \mathrm{N}=12$ & & $\Sigma \mathrm{X}=9.820$ & & $\Sigma Y=9.680$ & $\Sigma X Y=28.517$ & $\Sigma X^{2}=28.929$ & \multirow{5}{*}{98.95} \\
\hline \multirow{5}{*}{ Fosfestrol } & 1 & 3 & 0.969 & 0.954 & 1.898 & 0.944 & 0.901 & 0.910 & \\
\hline & 2 & 3 & 0.969 & 1.908 & 2.842 & 1.888 & 3.602 & 3.641 & \\
\hline & 3 & 3 & 0.969 & 2.862 & 3.786 & 2.832 & 8.105 & 8.191 & \\
\hline & 4 & 3 & 0.969 & 3.816 & 4.730 & 3.776 & 14.409 & 14.562 & \\
\hline & & $\Sigma \mathrm{N}=12$ & & $\Sigma X=9.540$ & & $\Sigma Y=9.440$ & $\Sigma X Y=27.017$ & $\Sigma X^{2}=27.304$ & \multirow{5}{*}{98.92} \\
\hline \multirow{5}{*}{ Letrozole } & 1 & 3 & 0.984 & 0.923 & 1.847 & 0.913 & 0.843 & 0.852 & \\
\hline & 2 & 3 & 0.984 & 1.846 & 2.760 & 1.826 & 3.371 & 3.408 & \\
\hline & 3 & 3 & 0.984 & 2.769 & 3.673 & 2.739 & 7.584 & 7.667 & \\
\hline & 4 & 3 & 0.984 & 3.692 & 4.586 & 3.652 & 13.483 & 13.631 & \\
\hline & & $\Sigma \mathrm{N}=12$ & & $\Sigma X=9.230$ & & $\Sigma Y=9.130$ & $\Sigma X Y=25.281$ & $\Sigma X^{2}=25.558$ & \multirow{5}{*}{98.82} \\
\hline \multirow{5}{*}{ Mitomycin } & 1 & 3 & 0.991 & 0.986 & 1.953 & 0.972 & 0.958 & 0.972 & \\
\hline & 2 & 3 & 0.991 & 1.964 & 2.925 & 1.944 & 3.818 & 3.857 & \\
\hline & 3 & 3 & 0.991 & 2.946 & 3.897 & 2.916 & 8.591 & 8.679 & \\
\hline & 4 & 3 & 0.991 & 3.944 & 4.869 & 3.888 & 15.334 & 15.555 & \\
\hline & & $\Sigma \mathrm{N}=12$ & & $\Sigma X=9.860$ & & $\Sigma Y=9.720$ & $\Sigma X Y=28.701$ & $\Sigma X^{2}=29.063$ & \\
\hline
\end{tabular}

$$
\text { Recovery }(\%)=\frac{\mathrm{N}(\Sigma X \mathrm{Y})-(\Sigma \mathrm{X})(\Sigma \mathrm{Y})}{\mathrm{N}\left(\Sigma \mathrm{x}^{2}\right)-(\Sigma \mathrm{X})^{2}} \times 100
$$

where, $\mathrm{N}=$ total number of observations, $\mathrm{X}=$ amount of drug added, $\mathrm{Y}=$ amount of drug obtained by calculation.

\section{ACKNOWLEDGEMENTS}

The authors are thankful to Cipla Pharmaceutical Ltd. and Panjon Pharmaceutical Ltd. Indore, India for providing the pure form of compounds as gift samples. The authors are also thankful to UGC, New Delhi, India for providing the financial assistance to carry out this work.

\section{REFERENCES}

1. M.J. Miller, Drugs, 20, 122 (1980).

2. Y.-C. Cheng and H.L. Po, Acta Neurol. Taiwanica, 20, 262 (2011).

3. C. Cantisani, S. Ricci, T. Grieco, G. Paolino, V. Faina, E. Silvestri and S. Calvieri, BioMed Res. Int., Article ID 151509 (2013); https://doi.org/10.1155/2013/151509.

4. I.E. Smith and M. Dowsett, N. Engl. J. Med., 348, 2431 (2003); https://doi.org/10.1056/NEJMra023246.

5. J.A. Orellana, N. Palacios-Prado and J.C. Saez, Toxicol. Appl. Pharmacol., 213, 187 (2006);

https://doi.org/10.1016/j.taap.2005.10.011.

6. A.R. Williams and P. Whelan, Prostate Cancer Prostatic Dis., 1, 204 (1998),

7. S. Boussios, G. Pentheroudakis, K. Katsanos and N. Pavlidisa, Ann Gastroenterol., 25, 106 (2012).
8. A.A.-M. Abdel-Hafez, Bioorg. Med. Chem., 10, 2297 (2002); https://doi.org/10.1016/S0968-0896(02)00047-0.

9. K. Pluta, B. Morak-Mlodawska and M. Jelen, Eur. J. Med. Chem., 46, 3179 (2011); https://doi.org/10.1016/j.ejmech.2011.05.013.

10. H.T. Akçay and R. Bayrak, Spectrochim. Acta A Mol. Biomol. Spectrosc., 122, 142 (2014); https://doi.org/10.1016/j.saa.2013.11.028.

11. A. Paci, L. Mercier and P. Bourget, J. Pharm. Biomed. Anal., 30, 1603 (2003); https://doi.org/10.1016/S0731-7085(02)00541-1.

12. P. Jandera and J. Churácek, J. Chromatogr. A, 98, 1 (1974); https://doi.org/10.1016/S0021-9673(00)84780-9.

13. V.K. Gupta, R. Jain, K. Radhapyari, N. Jadon and S. Agarwal, Anal. Biochem., 408, 179 (2011); https://doi.org/10.1016/j.ab.2010.09.027.

14. J.M. Calatayud and L.L.Zamora, Ref. Mod. Chem.Mol. Sci. Chem. Eng. (2013); https://doi.org/10.1016/B978-0-12-409547-2.00507-2.

15. N. Lewen, J. Pharm. Biomed. Anal., 55, 653 (2011); https://doi.org/10.1016/j.jpba.2010.11.030.

16. R. Zeleny, F. Ulberth, P. Gowik, J. Polzer, L.A. van Ginkel and H. Emons, Trends Analyt. Chem., 25, 927 (2006); https://doi.org/10.1016/j.trac.2006.04.011.

17. E.J. Corey and M. Ishiguro, Tetrahedron Lett., 20, 2745 (1979); https://doi.org/10.1016/S0040-4039(01)86404-2.

18. I.C. Shukla and V.N. Pathak, Indian J. Pharm. Sci., 44, 107 (1982).

19. I.C. Shukla, V. Kumar, B.K. Singh, O.P. Yadav and S. Kumar, Int. J. Pharma Bio. Sci., 2, 131 (2010).

20. Indian Pharmacopeia, The Controller of the Publication, Ghaziabad, India, vol. 2, p. 921, 1289 and 1605 (2007). 